\title{
Machine learning analysis of exome trios to contrast the genomic architecture of autism and schizophrenia
}

Sameer Sardaar ${ }^{1 \dagger}$, Bill Qi $^{1 \dagger}$, Alexandre Dionne-Laporte ${ }^{2}$, Guy. A. Rouleau ${ }^{1,2}$, Reihaneh Rabbany $y^{3,4}$ and Yannis J. Trakadis ${ }^{1,5^{*}}$ (D)

\begin{abstract}
Background: Machine learning (ML) algorithms and methods offer great tools to analyze large complex genomic datasets. Our goal was to compare the genomic architecture of schizophrenia (SCZ) and autism spectrum disorder (ASD) using ML.

Methods: In this paper, we used regularized gradient boosted machines to analyze whole-exome sequencing (WES) data from individuals SCZ and ASD in order to identify important distinguishing genetic features. We further demonstrated a method of gene clustering to highlight which subsets of genes identified by the ML algorithm are mutated concurrently in affected individuals and are central to each disease (i.e., ASD vs. SCZ "hub" genes).

Results: In summary, after correcting for population structure, we found that SCZ and ASD cases could be successfully separated based on genetic information, with 86-88\% accuracy on the testing dataset. Through bioinformatic analysis, we explored if combinations of genes concurrently mutated in patients with the same condition ("hub" genes) belong to specific pathways. Several themes were found to be associated with ASD, including calcium ion transmembrane transport, immune system/inflammation, synapse organization, and retinoid metabolic process. Moreover, ion transmembrane transport, neurotransmitter transport, and microtubule/ cytoskeleton processes were highlighted for SCZ.

Conclusions: Our manuscript introduces a novel comparative approach for studying the genetic architecture of genetically related diseases with complex inheritance and highlights genetic similarities and differences between ASD and SCZ.
\end{abstract}

Keywords: Genomic, Machine learning, Unsupervised clustering, Autism spectrum disorder, Schizophrenia

\section{Background}

Autism spectrum disorder (ASD) is a neurodevelopmental disorder characterized by significant impairments in social communication and interaction, as well as by abnormal repetitive behaviors, interests, or activities (Diagnostic and Statistical Manual of Mental Disorders (DSM)-5, 2013). The heritability of ASD has been estimated to be around 70$90 \%$, suggesting that genetic factors contribute largely to the

\footnotetext{
* Correspondence: yannis.trakadis@mcgill.ca

${ }^{+}$Sameer Sardaar and Bill Qi contributed equally to this work.

'Department of Human Genetics, McGill University, Montreal, QC, Canada

${ }^{5}$ Department of Medical Genetics, McGill University Health Center Room

A04.3140, Montreal Children's Hospital,1001 Boul. Décarie, H4A 3J1 Montreal,

Quebec, Canada

Full list of author information is available at the end of the article
}

ASD phenotype [1]. Genome-wide sequencing analyses have revealed that a large number (100 to 1000) of susceptibility genes are associated with ASD [2-4]. Recent studies showed that de novo mutations (DNM) have a significant role in ASD [3, 5-7], and estimated that around $30 \%$ of simplex ASD cases result from DNMs [3].

Schizophrenia (SCZ) is a neuropsychiatric disorder characterized by distorted perception, emotion, and cognition. It can also be characterized by negative symptoms, such as anhedonia, blunting of affect, or poverty of speech and thought (DSM-5, 2013). Similar to ASD, SCZ has high heritability, estimated to be around $80-85 \%$, yet, much of it is not fully understood [8]. Recent studies have highlighted a role for common single nucleotide polymorphisms (SNPs) in SCZ 
[9-12]. Moreover, like ASD, SCZ cases are enriched in de novo single nucleotide variants (SNVs) [13, 14].

In summary, both SCZ and ASD clearly have a strong genetic component in their etiopathology; however, linkage analysis and genome-wide associations have had limited success and replicability in identifying significant genes in these complex disorders [15-19]. The lack of success is thought to be due to ASD and SCZ having polygenic and multifactorial inheritance where, unlike Mendelian disorders, each susceptibility gene increases one's predisposition to the disease in combination with other genes. The involvement of many genes (in different combinations for each patient) and environmental factors makes it difficult to identify the specific genetic risk factors predisposing a given patient to ASD or SCZ.

Machine learning (ML) or statistical learning (SL) algorithms aim to learn and understand complex highdimensional data. These learning algorithms can be divided into two broad categories: supervised learning and unsupervised learning [20-22]. Our group recently applied supervised ML to rare, predicted functional variants from whole-exome sequencing (WES) data of a SCZ case-control dataset $(n=5090) .70 \%$ of the data was used to train the ML algorithm and $30 \%(n=1526)$ to evaluate its performance, showing encouraging results (86\% accuracy, AUC: 0.95) [23]. Studies based on supervised learning, like the one just mentioned, are focused on learning from input-to-output labeled data where a model is trained to learn the best function or map from input variables of data instances to their labels. In contrast, unsupervised learning algorithms seek to discover useful underlying patterns in a dataset without relying on labels. For instance, a recent publication using unsupervised learning illustrated how WES data could be used to identify patient subtypes of patients with major depressive disorder (MDD) [24].

Several studies have shown the effectiveness of supervised learning methods in distinguishing between overlapping medical conditions. For example, they have been used to distinguish between age-related cognitive decline and dementias based on neurocognitive tests [25]. Further, they have also been successfully used to distinguish and study different cancer types based on gene expressions [26, 27] and DNA methylation patterns [28].

Overlapping genetic factors conferring risk to both SCZ and ASD have been identified suggesting shared biological pathways [29]. Our hypothesis is that ML methods can help us advance our understanding of the genomic architecture of ASD and SCZ by contrasting exome data from patients with these two conditions. Analyzing data of individuals affected with two different conditions with high heritability, complex inheritance, and evidence for overlapping genetic features using supervised learning may have some advantages. For example, in our above-mentioned SCZ case-control study, some unaffected individuals may also be genetically at high risk for SCZ but not have been exposed to adequate environmental risk factors, complicating the analysis. When comparing individuals with ASD and SCZ, given they are all affected, this is not an issue anymore. The first objective of our study is to explore whether SCZ and ASD patients can be distinguished based solely on supervised learning analysis of the genetic information from their WES data. Our second objective is to analyze the genetic features prioritized by the supervised learning algorithm, using unsupervised clustering, to identify central hub genes in the genetic architecture for SCZ and ASD.

\section{Materials}

Whole-exome data sources and annotation Schizophrenia WES data (dbGaP trios)

This dataset is available in the dbGaP (study phs000687.v1.p1). The samples in this dataset were collected from the University Hospital Alexander in Sofia, Bulgaria. Individuals with intellectual disability were excluded. Unrelated families with parents who did not have schizophrenia participated in the original study. Overall, 598 trios were included in our analysis.

\section{Autism WES data (NDAR trios)}

The data for 2392 families with ASD were obtained from NDAR (doi: https://doi.org/10.15154/1169318; doi: https://doi.org/10.15154/1169195). The original sequencing data is of families in the Simons Simplex Collection [30]. The proband had to: 1 ) be at least 36 months of age, 2) have a nonverbal IQ or nonverbal mental age of 24 months for children aged between 36 and 83 months, or 30 months for children aged 84 months and above, 3) not have a known genetic disorder, and 4) not have extensive birth complications such as prematurity and cerebral palsy. Moreover, one of the requirements for participation in the study was that both biological parents had to be willing to participate and that they should not have ASD.

\section{Summary of variant filtering criteria}

Filtering was run through the rows of variants in each dataset so that only variants that met the following criteria were included in our analysis.

We selected for coding variant types annotated as "frameshift_deletion", "frameshift_insertion", "frameshift_ substitution", "nonsynonymous_SNV", "stopgain", or "stoploss," and variant functional types annotated as "exonic", "exonic_splicing", or "intronic_splicing." Furthermore, the selected variants had a minor allele frequency (MAF) equal to or less than 0.01 . Lastly, on a per-individual basis, 
for variants to be called they needed to have a minimum number of 4 variant reads, a minimum depth of sequencing of 10 reads, and a minimum genotype quality of 90 .

The selected variants were then arranged in a tabular format, where each row corresponded to a different individual. The clinical status (ASD vs SCZ) for each individual was denoted in the first column, while the variants meeting our criteria for each individual were denoted as separate columns, with values of 0,1 or 2 in the corresponding cells indicating wildtype, heterozygous, and homozygous status for each selected variant for the respective individual.

\section{Methods}

\section{Population stratification adjustment}

A major confounder in the analysis of cross-origin datasets like the ones we are using is the population stratification due to differences in ancestry. Due to population structure, the ML algorithm could focus on SNVs unrelated to the disease, which are specific to the population from which the affected individuals originate. Our focusing on rare variants minimizes the impact of differences in population structure between the two datasets. However, to formally address this possibility, we implemented a well-established population stratification correction method for genome-wide data [Eigenstrat] [31]. Eigenstrat is based on the adjustment of the original SNVs data based on any population structure discovered using principal components analysis. We applied this approach to adjust for population differences between the ASD (NDAR trios) and the SCZ (dbGaP trios) datasets. To remove the population structure from our dataset, we used the top 4 axes of variation from Eigenstrat that were significant. This is expected to account for most of the population structure. Then we regressed each SNV or feature of our dataset on the four axes of variation and took its residuals to be the adjusted SNV values of our adjusted dataset that corrects for population structure. We adjusted the phenotype values in a similar fashion. Lastly, each adjusted genotype and phenotype value was rounded to the nearest whole number to estimate the nearest adjusted genotype and phenotype. As a result, the original binary class of ASD and SCZ was converted to integer values, which we then capped to a range of -4 to +4 as only one adjusted instance fell outside this range.

This dataset has the adjusted genotype values of each SNV arranged in columns for each row of patient sample and will be referred to as the $S N V$-based data. We also converted the adjusted SNVs datasets into "genelevel SNV counts" by summing together all adjusted SNVs values located in the same gene of any given patient. This dataset has the sums for each gene arranged in columns for each row of patient sample and is referred to as the gene-based data.

\section{Algorithm selection}

Many powerful ML algorithms render themselves uninterpretable, making it difficult to understand their decision-making process. Trying to balance interpretability with model performance, we used a more interpretable state of the art ML algorithm: regularized gradient boosted machine (GBM) (XGBoost implementation) [32], which we also demonstrated as an effective algorithm in our previous study [23].

Regularized GBM is state of the art and has been proved successful in a wide range of tasks. Its highly regularized methodology of feature selection and ranking of features based on their relative importance in making accurate predictions made it a great candidate for our study. Of note, a regularized algorithm penalizes itself for complexity, and thus uses only features that are relevant and brings more intelligence to its architecture than complexity. In our study, this means using only genes that have high predictive power in combination with other genes, and discard the less informative ones, thereby reducing the number of candidate genes.

\section{Training the boosted regression trees models}

Since the population structure adjusted datasets following the Eigenstrat methodology have continuous phenotype labels, we trained the boosted regression trees variant of GBM to predict the continuous label values of ASD and SCZ cases based on the SNV-based data and the gene-based data. Since the focus of this analysis is to classify patients as either ASD or SCZ, we framed the regression problem as a classification to allow for measurement of the prediction accuracy. We performed the following mapping of the continuous predicted value to the binary classes. Since the adjusted phenotype values for ASD cases all had values of 1 or greater, and the adjusted phenotype values for SCZ all had values of -1 or lower, any prediction above 0 was mapped to a prediction of ASD class and any prediction below 0 was mapped to a prediction of SCZ class.

Given our ASD and SCZ datasets contain an unbalanced number of individuals, we decided to use a balanced approach by selecting an equal number of ASD and SCZ cases. This change ensured that accuracy would be a good measure of model performance. To this end, the first 598 samples were selected from the ASD cases to balance the two datasets. We trained and finetuned the boosted regression trees using $70 \%$ of the data (419 ASD vs. 419 SCZ samples) as a training and validation dataset. We then inspected the best performing model on the remaining, previously unseen, $30 \%$ of the data (test dataset; 179 ASD vs. 179 SCZ samples). The SNVs used by the $S N V$-based model were extracted and mapped to their corresponding genes to get the list of 
the most important genes. The most important genes used by the best gene-based model were also extracted.

In addition to the 70:30 split for evaluation, we also assessed the performance of a five-fold cross-validation using the whole dataset (598 ASD vs. 598 SCZ samples) to provide a comprehensive validation of the algorithm.

\section{Identification of genes central to ASD and SCZ}

To find which genes are important to SCZ or ASD, and which of these genes appear to be mutated concurrently in affected individuals, a novel unsupervised clustering analysis was performed. The genes identified by the 1) $S N V$-based algorithm and 2) gene-based algorithm were compared, and the ones identified by both algorithms (the overlapping ML list of genes) were used for the subsequent analyses.

To identify the (networks of) genes important to SCZ, hierarchical clustering was performed for the overlapping $M L$ list of genes, using only the SCZ cases and the genebased dataset. The Jaccard coefficient was used as the similarity measure for clustering the genes. The Jaccard coefficient between any two genes was calculated as the number of shared SCZ cases having an SNV count value greater than 0 in both genes divided by the number of SCZ cases having an SNV count value greater than 0 in either gene. Gene distances were derived as one minus the Jaccard coefficient. Hierarchical clustering is performed based on the distances using Ward's linkage method [33], which recursively joins elements and/or clusters to form new clusters while minimizing the increase in the variance of the new cluster. Lastly, a dendrogram showing clusters of similar genes based on the distance metric and linkage method was created. To determine the most important cluster of genes for SCZ, we applied the following approach.

For each gene cluster identified, the number of genes was counted (a). Similarly, the number of unique SCZ cases carrying a genetic change in at least one of these genes was determined (b). This number (b) represents the number of $\mathrm{SCZ}$ cases having a genetic variation in at least one of the genes in a given cluster. Then, by dividing (b) over (a) a ratio, specific for each cluster, was calculated. The cluster with the highest ratio was selected

Table 1 Performance of different approaches (algorithms) on test data

\begin{tabular}{|c|c|c|c|c|c|c|}
\hline Method & Accuracy & Precision & Recall & NIR & $\begin{array}{l}P \text {-value (Acc > } \\
\text { NIR) }\end{array}$ & $95 \% \mathrm{Cl}$ \\
\hline $\begin{array}{l}\text { SNV- } \\
\text { based }\end{array}$ & 0.86 & 0.73 & 0.98 & 0.63 & $<4.97 \mathrm{e}-22$ & $\begin{array}{l}(0.82 \\
0.89)\end{array}$ \\
\hline $\begin{array}{l}\text { Gene- } \\
\text { based }\end{array}$ & 0.88 & 0.80 & 0.96 & 0.58 & $<3.09 \mathrm{e}-36$ & $\begin{array}{l}(0.85 \\
0.92)\end{array}$ \\
\hline
\end{tabular}

The performance between the two algorithms trained to distinguish ASD cases from SCZ cases is measured on a previously unseen test dataset. The accuracy is a measure of the number of correctly predicted samples divided by the total number of samples

Acc Accuracy, NIR No information rate, Cl Confidence interval
Table 2 Performance of SNV and Gene-based approaches using five-fold cross validation

\begin{tabular}{|c|c|c|c|c|c|c|}
\hline Method & Accuracy & Precision & Recall & NIR & $\begin{array}{l}P \text {-value (Acc > } \\
\text { NIR) }\end{array}$ & $95 \% \mathrm{Cl}$ \\
\hline $\begin{array}{l}\text { SNV- } \\
\text { based }\end{array}$ & 0.88 & 0.78 & 0.97 & 0.59 & $<2.2 \mathrm{e}-16$ & $\begin{array}{l}(0.86 \\
0.90)\end{array}$ \\
\hline $\begin{array}{l}\text { Gene- } \\
\text { based }\end{array}$ & 0.88 & 0.81 & 0.95 & 0.57 & $<2.2 \mathrm{e}-16$ & $\begin{array}{l}(0.86 \\
0.90)\end{array}$ \\
\hline
\end{tabular}

The performance between the two algorithms trained to distinguish ASD cases from SCZ cases is measured using five-fold cross validation. All performance metrics are the average of the five cross validation folds Acc Accuracy, NIR No information rate, $\mathrm{Cl}$ Confidence interval

as the one containing genes central to SCZ, as it involved genes highly mutated, in different combinations, among the highest proportion of SCZ patients in our dataset.

The same analysis above was then repeated separately based on ASD cases to obtain the genes central to ASD.

\section{Analysis software}

The "xgboost" (version 0.90.0.1) package [34] for R was used as the implementation of the XGBoost algorithm. The "scipy" (version 1.0.1) package [35] for Python was used for the hierarchical clustering analyses.

\section{Results}

For our boosted regression trees models, we obtained an accuracy of $86 \%$ for the $S N V$-based model and $88 \%$ for the gene-based model. Detailed metrics of model performance are listed in Table 1. A five-fold cross validation was also performed to provide additional validation. Overall, the average validation accuracy over all five folds was $88 \%$ for both the $S N V$-based model and gene-based model (Table 2). The performance over cross-validation is consistent with the results from the single-fold training-validating with independent testing approach mentioned above.

The ten most important genes from the gene-based model and the $S N V$-based approach (including the actual SNV in parenthesis) are shown in Table 3. The SNV-based model utilized 322 SNVs, located in 313 unique genes. The gene-based model utilized 1845 genes. Combining the top 10 genes from both approaches yielded a total list of 16 genes (Supplemental Table 1), with an overlap of 4 genes including the top 2: SARM1 and QRICH2, and PCLO and PRPF31. Overall, out of all the genes used by both models, 151 genes were overlapping (Supplemental Table 2).

Clustering of these 151 overlapping genes based on SCZ cases revealed three clusters of genes. Out of the three clusters, cluster 2 showed the highest ratio (7.55) of SCZ cases per cluster gene. Overall, 84.62\% (506/598) of SCZ cases in our dataset had a genetic change in at least one of the genes in SCZ cluster 2, which is 
Table 3 Top 10 important genes from SNV-based and genebased models

\begin{tabular}{ll}
\hline SNV-based approach (SNV rsID) & Gene-based approach \\
\hline SARM1 (rs71373646) & SARM1 \\
QRICH2 (rs6501878) & QRICH2 \\
AKAP1 (rs34535433) & PRPF31 \\
PCLO (rs77721383) & SEC24D \\
TSPO2 (rs147405274) & SCN4A \\
ABCC3 (rs11568605) & CACNA1S \\
KIF13A (rs41267712) & CDSN \\
FAN1 (rs150393409) & HERC2 \\
CCDC155 (rs201671744) & MUC16 \\
PRPF31 (rs199870856) & PCLO
\end{tabular}

Boosted regression trees models were trained to separate SCZ and ASD probands based on the population-structure-adjusted SNV-based and genebased datasets. The 10 most important genes from the gene-based model, but also from the SNV-based approach (including the actual SNV in parenthesis), are shown in this table. The table is ordered from most to least importance

composed of 67 genes (Fig. 1, Supplemental Table 3). Similarly, clustering of the 151 overlapping genes, from Supplemental Table 2, based on ASD cases, revealed two clusters of genes. The highest ratio of cases per gene was 15.5 from ASD cluster 2. Overall, 98.49\% (589/598) of ASD cases in our dataset had a genetic change in at least one of the genes in ASD cluster 2, which is composed of 38 , out of the 151 overlapping genes being targeted (Fig. 2, Supplemental Table 4).

\section{Discussion}

We have explored the genetic architecture of SCZ and ASD families through boosted regression trees (XGBoost) and clustering. Our focusing on rare variants minimizes the impact of differences in population structure between the two datasets. However, before performing any analyses, we also used the well-known Eigenstrat method to correct for any differences between datasets due to population structure. Overall, through boosted regression trees, we were able to find SNVs (and genes) which can distinguish between SCZ and ASD case status with accuracies of $88 \%$ for cross-validation and $85-90 \%$ on testing data (specifically, $86 \%$ for $S N V$-based method and $88 \%$ for gene-based method). To further study the important genes identified from the boosted regression trees, we hierarchically clustered the 151 genes identified from both algorithms (Supplemental Table 2) using only SCZ cases (and repeated the process for ASD). Our hypothesis was that some of the genes identified as part of the boosted regression trees approach might be important, central "hubs" for SCZ (and/or ASD). Through clustering of the 151 overlapping genes, based on the shared proportion of cases between genes, we were able to find groups of genes that were often mutated together in SCZ cases (and ASD cases, respectively).

Overall, we have demonstrated a novel approach for studying (comparing) the genetic architecture and pathophysiology of two diseases. Instead of using all SNVs from WES data, we first utilized a regularized machine learning approach optimized for large feature sets to identify the most important genes for separating the two groups (ASD and SCZ in this case). This step can potentially reduce the number of features by a magnitude or more to eliminate noise from additional features (SNVs and genes with no or little impact, in our case). We have demonstrated that boosted regression trees can separate SCZ and ASD patients based solely on their WES data. This highlights the role of ML in deciphering the genomic architecture of different diseases with shared genetics.

Next, we identified (networks of) genes that are important for each disease, through hierarchical clustering of genes based on the proportion of cases they shared. Although each of the 151 genes may contribute to SCZ or ASD to some extent, our focus was to find the central group of genes that plays an important role in the majority of our cases. Our clustering method highlighted these genes for SCZ (Fig. 1, Supplemental Table 3) and ASD (Fig. 2, Supplemental Table 4). The dendrograms created based on this approach denote genes mutated concurrently in affected individuals and thus provide information about the networks of genes that appear to be important for each of the diseases targeted. This approach can potentially help address the clinical heterogeneity of each disease. For example, after identifying the central genes for SCZ, these genes can be used to cluster SCZ patients and look for subgroups that could then be characterized based on genetics, clinical features, medication response, or disease progression.

Our bioinformatic analysis and literature review of the identified genes revealed multiple pathways and networks important to SCZ and/or ASD. Focusing on the top 10 genes identified by the two boosted regression trees approaches (Supplemental Table 1), we found that some of them already have evidence in the literature linking them to SCZ and/or ASD.

For example, KIF13A is a member of the kinesin superfamily proteins (KIFs), which are important for cellular transport and signal transduction [36]. KIF13A is located in a SCZ susceptibility region of chromosome $6 \mathrm{p} 23$. A recent study on mice lacking KIF13A reported elevated anxiety-related traits through a reduction in the serotonin $5 \mathrm{HT}(1 \mathrm{~A}) \mathrm{R}$ receptor transport and reduced expression of the receptor in neuroblastoma cells and hippocampal neurons [37]. Another study investigating the mechanism of endosomal recycling revealed that KIF13A interacts with the protein complex BLOC-1 and 


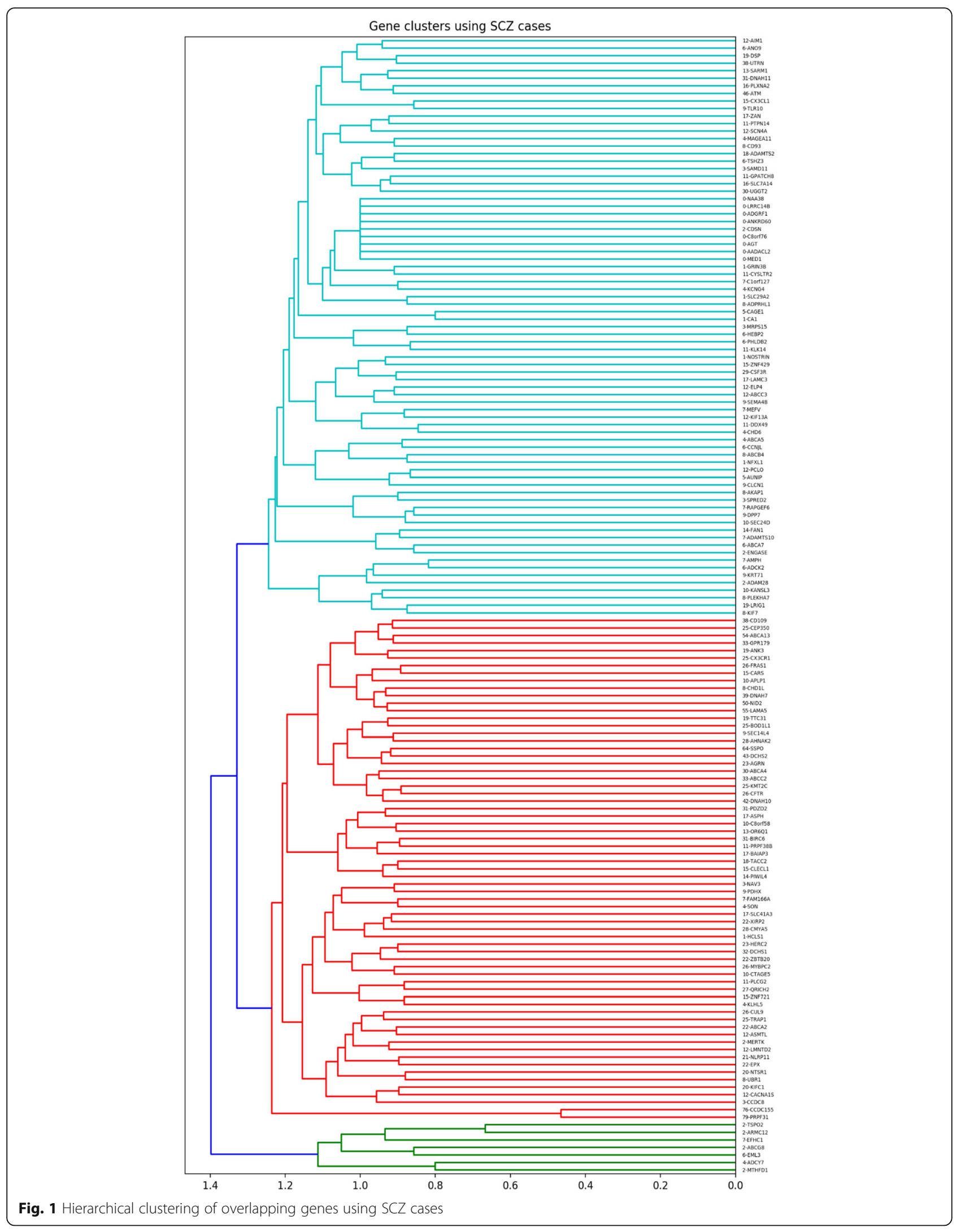




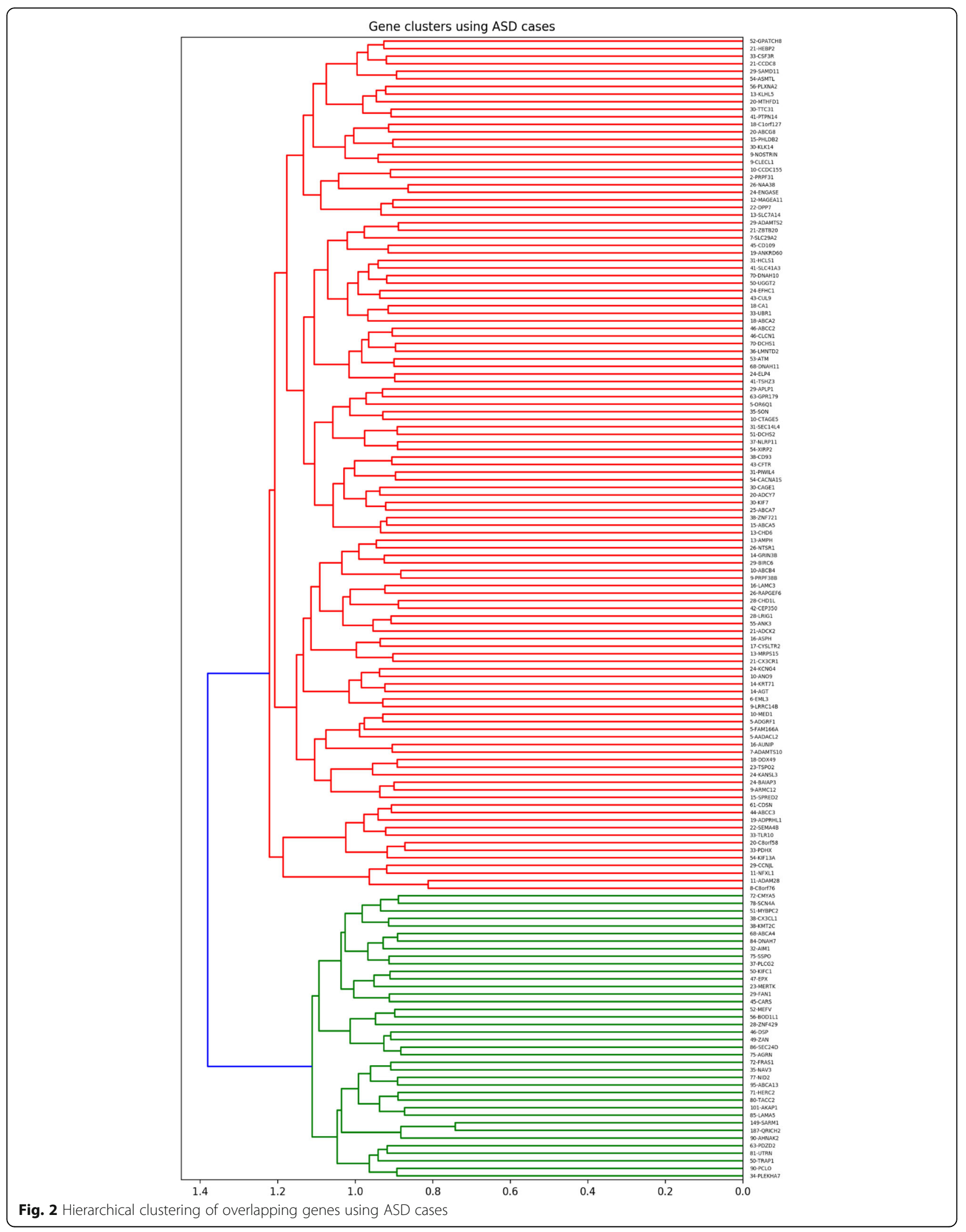


Annexin A2, and that dysfunction of these interactions may underly the pathophysiology of neurological defects associated with SCZ [38]. Of note, a rare disruption of another member of the KIFs, KIF17, could also lead to SCZ [39]. No evidence was found supporting the involvement of this gene in ASD.

Fanconi-associated nuclease 1 (FAN1), a DNA repair enzyme, is located in the chromosome 15q13.3 locus. A microdeletion in the locus, affecting FAN1 and six other genes, is associated with increased risk of both ASD and SCZ. Deletion of this region using mice models resulted in increased seizure susceptibility and ASD symptoms among other defects [40]. A study systematically searching for SCZ risk variants identified variants in FAN1, which were associated with both SCZ and ASD [41].

Literature review of the genes revealed evidence for both SCZ and ASD, which is consistent with the gene networks hypothesis of common underlying genetic drivers. At the same time, it is interesting to note that some of the genes we identified do not have a (clear) previous link to SCZ or ASD, suggesting that the approaches described in this manuscript can potentially yield new insights for the genetics of the conditions targeted.

Additionally, we conducted a bioinformatics analysis and literature review of the SCZ and ASD "hub" genes. Pathway enrichment analysis was performed using the ShinyGO tool v0.61 [42] based on the ASD "hub" genes (Supplemental Table 3) and SCZ "hub" genes (Supplemental Table 4) identified. Based on the pathway network plot generated with Gene Ontology (GO) biological processes meeting a false-discovery rate (FDR) less than 0.2 (Supplemental Figures 1 and 2), we identified several themes. For ASD, we identified the following themes: 1) calcium ion transmembrane transport, 2) immune system and inflammation, 3) cell projection, neuron maturation and synapse organization, 4) retinoid metabolic process, 5) actin-related processes, and 6) blood and platelet coagulation processes.

There is evidence that changes in calcium signaling may be associated with ASD [43-45]. Similarly, multiple studies support a link of immune dysfunction and inflammation to ASD [46-48], while strong evidence exists for a link with synaptic structures [49-52]. Upregulation of immune genes and downregulation of synaptic genes was observed in the postmortem brains of idiopathic ASD patients [53, 54]. Recent analyses in larger ASD cohorts of postmortem brain collections showed upregulation of immune-microglia and mitochondrial modules, and downregulation of neuronal and synaptic modules [55].

Furthermore, actin and microtubule processes are linked to ASD [56]. Also, alterations in actin dynamics by actinbinding proteins and calcium signaling messengers is associated with ASD [57]. In contrast to SCZ, ASD is associated with an increase in dendritic spine density in several areas of the brain [50], which is thought to be mainly regulated via postsynaptic actin filaments [57].

Some evidence also exists for a link of ASD to retinoid and retinoic acid metabolic processes [58, 59], as well as abnormalities in platelet and coagulation pathways [6062].

As illustrated above, several of the themes identified have evidence for a joint role in ASD. In support to this, in Fragile $\mathrm{X}$, a well-known syndrome associated with ASD, evidence has been published for all pathways mentioned above: from dysregulation of calcium signaling, synaptic structures, actin to inflammation, and changes in the retinoid and coagulation pathways [63-69].

For SCZ, our pathway enrichment analysis identified the following themes: 1) ion transmembrane transport/ neurotransmitter transport, 2) microtubule/cytoskeleton, 3) response to carbohydrates/glucose/hexose stimulus, and 4) kidney/renal system development. There is robust evidence in the literature for the role of neurotransmitters in SCZ [70, 71]. Moreover, recurrent evidence exists linking microtubules/cytoskeleton and SCZ [72-78]. There is not much evidence for kidney development and SCZ, but there have been studies showing that SCZ is associated with chronic kidney disease, even after controlling for demographic, behavioral, and medical risk factors $[79,80]$. Furthermore, a study found a polygenic signature differentiating SCZ from controls, which could also significantly differentiate type 2 diabetes patients from controls by predicting a glycemic control indicator, supporting a molecular commonality between SCZ and type 2 diabetes [81]. Of note, Glucose metabolism has been shown to be impaired in patients with first-episode SCZ [82] and in antipsychotic-naïve patients with psychosis [83].

\section{Conclusion}

We first showed that supervised learning can distinguish SCZ and ASD patients with high accuracy based solely on their rare SNVs in 151 genes. Through clustering analysis of these genes, we highlighted the important "hub" genes contributing to SCZ or ASD. Bioinformatic analysis revealed several biological themes associated with the "hub" genes of each disorder, including calcium ion transmembrane transport, immune system/inflammation, synapse organization, and retinoid metabolic process for ASD versus ion transmembrane transport, neurotransmitter transport, and microtubule/cytoskeleton processes for SCZ. Our findings demonstrate the usefulness of ML analysis of exome data in the study of the genetic architecture of distinct, yet genetically overlapping, diseases with complex inheritance. 


\section{Future directions}

In addition to rare SNV, common variants [84] and copy number variations (CNVs) also have support in the literature for a role in ASD $[85,86]$. Similarly, de novo CNVs $[13,14]$ and common variants have also been associated with SCZ $[87,88]$. The presence of CNVs contributing to these conditions suggests that it would be beneficial for future studies to focus on whole-genome sequencing (WGS) data, thus capturing both SNVs and CNVs, for $\mathrm{ML}$ analyses. Moreover, the presence of common variants as contributing factors to SCZ and ASD suggests that we should not only focus on rare variants but also factor in common variants in future ML analyses.

\section{Supplementary information}

Supplementary information accompanies this paper at https://doi.org/10. 1186/s12888-020-02503-5.

Additional file 1: Supplemental Table 1. Merged top 10 genes from the SNV-based model and top 10 genes from the gene-based model. Supplemental Table 2. Overlapping genes from the SNV-based model and the gene-based model. Supplemental Table 3. Cluster genes from gene clustering with SCZ patients. Supplemental Table 4. Cluster 2 genes from gene clustering with ASD patients.

Additional file 2: Supplemental Figure 1. Network plot of significant Gene Ontology biological processes for ASD. Supplemental Figure 2. Network plot of significant Gene Ontology biological processes for SCZ.

\section{Abbreviations}

ASD: Autism spectrum disorder; CNV: Copy number variation; DNM: De novo mutation; GBM: Gradient boosted machine; MAF: Minor allele frequency; ML: Machine learning; SCZ: Schizophrenia; SNP: Single nucleotide polymorphism; SNV: Single nucleotide variant; WES: Whole Exome Sequencing; WGS: Whole Genome Sequencing

\section{Acknowledgments}

We thank the authors and dbGaP for access to the WES data. We thank NDAR and the authors for access to the WES data. This manuscript reflects the views of the authors and may not reflect the opinions and views of the $\mathrm{NIH}$. We also thank Imane Bennani and Serena Mayar for helping perform the literature searches and collecting relevant articles.

\section{Authors' contributions}

$A D L$ and GR were in charge of the bioinformatic analysis of the variants. SS and $\mathrm{BQ}$ prioritized the variants of interest and performed all analyses, the literature review, and drafting of the manuscript, under the supervision of $Y T$ who conceived, designed, and coordinated the project. SS, BQ, and YT designed the original methodology. The machine learning methodology was verified by our collaborator RR from the McGill School of Computer Science, and Montreal Institute for Learning Algorithms. All authors reviewed and provided feedback on the manuscript. All authors have read and approved the manuscript.

\section{Funding}

Not applicable.

\section{Availability of data and materials}

The SCZ data used in the preparation of this manuscript were obtained from the Database of Genotypes and Phenotypes (dbGaP) after McGill IRB approval. Raw data used is available in study phs000473.v1.p1. The ASD dataset used in the preparation of this manuscript was obtained from the $\mathrm{NIH}$ supported National Database for Autism Research (NDAR). Raw data (including VCF files and additional data files) accessed and used in the preparation of this study is available from NDAR (doi: https://doi.org/10. 15154/1169318; doi: https://doi.org/10.15154/1169195).
Ethics approval and consent to participate

Not applicable.

\section{Consent for publication}

Not applicable.

\section{Competing interests}

The authors declare that they have no competing interests.

\section{Author details}

${ }^{1}$ Department of Human Genetics, McGill University, Montreal, QC, Canada. ${ }^{2}$ Department of Neurology and Neurosurgery, Montreal Neurological Institute, McGill University, Montreal, QC, Canada. ${ }^{3}$ School of Computer Science, McGill University, Montreal, QC, Canada. ${ }^{4}$ Montreal Institute for Learning Algorithms, Université de Montréal, Montreal, QC, Canada. ${ }^{5}$ Department of Medical Genetics, McGill University Health Center Room A04.3140, Montreal Children's Hospital,1001 Boul. Décarie, H4A 3J1 Montreal, Quebec, Canada.

Received: 18 November 2019 Accepted: 17 February 2020

Published online: 28 February 2020

\section{References}

1. Schaefer GB. Clinical genetic aspects of ASD spectrum disorders. Int J Mol Sci. 2016;17(2):180

2. Griswold AJ, Dueker ND, Van Booven D, Rantus JA, Jaworski JM, Slifer SH, et al. Targeted massively parallel sequencing of autism spectrum disorderassociated genes in a case control cohort reveals rare loss-of-function risk variants. Mol Autism. 2015;6:43.

3. Iossifov I, O'Roak BJ, Sanders SJ, Ronemus M, Krumm N, Levy D, et al. The contribution of de novo coding mutations to autism spectrum disorder. Nature. 2014;515(7526):216-21.

4. Iossifov I, Ronemus M, Levy D, Wang Z, Hakker I, Rosenbaum J, et al. De novo gene disruptions in children on the autistic spectrum. Neuron. 2012; 74(2):285-99.

5. Awadalla P, Gauthier J, Myers RA, Casals F, Hamdan FF, Griffing AR, et al. Direct measure of the de novo mutation rate in autism and schizophrenia cohorts. Am J Hum Genet. 2010;87(3):316-24.

6. Ronemus $M$, lossifov I, Levy $D$, Wigler $M$. The role of de novo mutations in the genetics of autism spectrum disorders. Nat Rev Genet. 2014;15:133.

7. Turner TN, Coe BP, Dickel DE, Hoekzema K, Nelson BJ, Zody MC, et al. Genomic patterns of de novo mutation in simplex autism. Cell. 2017;171(3): 710-22.e12.

8. Sullivan PF, Kendler KS, Neale MC. Schizophrenia as a complex trait: evidence from a meta-analysis of twin studies. Arch Gen Psychiatry. 2003; 60(12):1187-92.

9. Ripke S, O'Dushlaine C, Chambert K, Moran JL, Kahler AK, Akterin S, et al. Genome-wide association analysis identifies 13 new risk loci for schizophrenia. Nat Genet. 2013;45(10):1150-9.

10. Consortium SPG-WASG. Genome-wide association study identifies five new schizophrenia loci. Nat Genet. 2011;43(10):969-76.

11. Shi J, Levinson DF, Duan J, Sanders AR, Zheng Y, Pe'er I, et al. Common variants on chromosome 6p22.1 are associated with schizophrenia. Nature. 2009;460(7256):753-7.

12. Stefansson H, Ophoff RA, Steinberg S, Andreassen OA, Cichon S, Rujescu D, et al. Common variants conferring risk of schizophrenia. Nature. 2009; 460(7256):744-7.

13. McCarthy SE, Gillis J, Kramer M, Lihm J, Yoon S, Berstein Y, et al. De novo mutations in schizophrenia implicate chromatin remodeling and support a genetic overlap with autism and intellectual disability. Mol Psychiatry. 2014; 19:652.

14. Xu B, Roos JL, Levy S, van Rensburg EJ, Gogos JA, Karayiorgou M. Strong association of de novo copy number mutations with sporadic schizophrenia. Nat Genet. 2008;40(7):880-5.

15. De Rubeis S, Buxbaum JD. Genetics and genomics of autism spectrum disorder: embracing complexity. Hum Mol Genet. 2015:24(R1):R24-31.

16. Altmuller J, Palmer LJ, Fischer G, Scherb H, Wjst M. Genomewide scans of complex human diseases: true linkage is hard to find. Am J Hum Genet. 2001;69(5):936-50.

17. Henriksen MG, Nordgaard J, Jansson LB. Genetics of schizophrenia: overview of methods, findings and limitations. Front Hum Neurosci. 2017;11:322. 
18. Ramaswami $\mathrm{G}$, Geschwind $\mathrm{DH}$. Genetics of autism spectrum disorder. Handb Clin Neurol. 2018;147:321-9. https://doi.org/10.1016/B978-0-44463233-3.00021-X

19. Risch NJ. Searching for genetic determinants in the new millennium. Nature. 2000;405:847.

20. Deo RC. Machine learning in medicine. Circulation. 2015;132(20):1920-30.

21. Shalev-Shwartz S, Ben-David S. Understanding machine learning: from theory to algorithms. Cambridge: Cambridge University Press; 2014.

22. Friedman J, Hastie T, Tibshirani R. The elements of statistical learning: Springer series in statistics. New York; 2001. https://link.springer.com/book/1 0.1007/978-0-387-84858-7.

23. Trakadis YJ, Sardaar S, Chen A, Fulginiti V, Krishnan A. Machine learning in schizophrenia genomics, a case-control study using 5,090 exomes. Am J Med Genet B Neuropsychiatr Genet. 2019;180(2):103-12. https://doi.org/10. 1002/ajmg.b.32638. Epub 2018 Apr 28.

24. Yu C, Arcos-Burgos M, Licinio J, Wong ML. A latent genetic subtype of major depression identified by whole-exome genotyping data in a MexicanAmerican cohort. Transl Psychiatry. 2017;7(5):e1134

25. Er F, Iscen P, Sahin S, Cinar N, Karsidag S, Goularas D. Distinguishing agerelated cognitive decline from dementias: a study based on machine learning algorithms. J Clin Neurosci. 2017:42:186-92.

26. Gao F, Wang W, Tan M, Zhu L, Zhang Y, Fessler E, et al. DeepCC: a novel deep learning-based framework for cancer molecular subtype classification. Oncogenesis. 2019;8(9):44.

27. Sun Y, Zhu S, Ma K, Liu W, Yue Y, Hu G, et al. Identification of 12 cancer types through genome deep learning. Sci Rep. 2019;9(1):17256.

28. Jurmeister P, Schöler A, Arnold A, Klauschen F, Lenze D, Hummel M, et al. DNA methylation profiling reliably distinguishes pulmonary enteric adenocarcinoma from metastatic colorectal cancer. Mod Pathol. 2019;32(6): 855-65

29. Carroll LS, Owen MJ. Genetic overlap between autism, schizophrenia and bipolar disorder. Genome Med. 2009;1(10):102

30. Fischbach GD, Lord C. The Simons simplex collection: a resource for identification of autism genetic risk factors. Neuron. 2010;68(2):192-5.

31. Price AL, Patterson NJ, Plenge RM, Weinblatt ME, Shadick NA, Reich D. Principal components analysis corrects for stratification in genome-wide association studies. Nat Genet. 2006;38(8):904-9.

32. Chen T, Guestrin C. XGBoost: a scalable tree boosting system. In: Proceedings of the 22nd ACM SIGKDD international conference on knowledge discovery and data mining. San Francisco: ACM; 2016. p. 785-94.

33. Ward JH Jr. Hierarchical grouping to optimize an objective function. J Am Stat Assoc. 1963;58(301):236-44

34. Chen T, He T, Benesty M, Khotilovich V. Package 'xgboost'. R version 090; 2019

35. Virtanen P, Gommers R, Oliphant TE, Haberland M, Reddy T, Cournapeau D, et al. SciPy 1.0--fundamental algorithms for scientific computing in Python. 2019. arXiv preprint arXiv:190710121.

36. Hirokawa N, Tanaka Y. Kinesin superfamily proteins (KIFs): various functions and their relevance for important phenomena in life and diseases. Exp Cell Res. 2015:334(1):16-25.

37. Zhou R, Niwa S, Guillaud L, Tong Y, Hirokawa N. A molecular motor, KIF13A, controls anxiety by transporting the serotonin type $1 \mathrm{~A}$ receptor. Cell Rep. 2013;3(2):509-19.

38. Delevoye C, Heiligenstein X, Ripoll L, Gilles-Marsens F, Dennis MK, Linares RA, et al. BLOC-1 brings together the actin and microtubule cytoskeletons to generate recycling endosomes. Curr Biol. 2016;26(1):1-13.

39. Tarabeux J, Champagne N, Brustein E, Hamdan FF, Gauthier J, Lapointe M, et al. De novo truncating mutation in Kinesin 17 associated with schizophrenia. Biol Psychiatry. 2010;68(7):649-56.

40. Forsingdal $A$, Fejgin $K$, Nielsen V, Werge T, Nielsen J. 15q13.3 homozygous knockout mouse model display epilepsy-, autism- and schizophrenia-related phenotypes. Transl Psychiatry. 2016;6(7):e860.

41. Ionita-Laza I, Xu B, Makarov V, Buxbaum JD, Roos JL, Gogos JA, et al. Scan statistic-based analysis of exome sequencing data identifies FAN1 at 15 q13. 3 as a susceptibility gene for schizophrenia and autism. Proc Natl Acad Sci U S A. 2014;111(1):343-8.

42. Ge SX, Jung D. ShinyGO: a graphical enrichment tool for ani-mals and plants: bioRxiv; 2018.

43. Breitenkamp AF, Matthes J, Herzig S. Voltage-gated calcium channels and autism Spectrum disorders. Curr Mol Pharmacol. 2015;8(2):123-32.
44. Eyles DW, Burne TH, McGrath JJ. Vitamin D, effects on brain development, adult brain function and the links between low levels of vitamin $D$ and neuropsychiatric disease. Front Neuroendocrinol. 2013;34(1):47-64.

45. Pasca SP, Portmann T, Voineagu I, Yazawa M, Shcheglovitov A, Pasca AM, et al. Using iPSC-derived neurons to uncover cellular phenotypes associated with Timothy syndrome. Nat Med. 2011;17(12):1657-62.

46. Gladysz D, Krzywdzinska A, Hozyasz KK. Immune abnormalities in autism spectrum disorder-could they hold promise for causative treatment? Mol Neurobiol. 2018:55(8):6387-435.

47. Hughes HK, Mills Ko E, Rose D, Ashwood P. Immune dysfunction and autoimmunity as pathological mechanisms in autism spectrum disorders. Front Cell Neurosci. 2018;12:405.

48. Kocovska E, Gaughran F, Krivoy A, Meier UC. Vitamin-D deficiency as a potential environmental risk factor in multiple sclerosis, schizophrenia, and autism. Front Psychiatry. 2017:8:47.

49. Guang S, Pang N, Deng $X$, Yang L, He F, Wu L, et al. Synaptopathology involved in autism spectrum disorder. Front Cell Neurosci. 2018;12:470.

50. Hutsler JJ, Zhang H. Increased dendritic spine densities on cortical projection neurons in autism spectrum disorders. Brain Res. 2010;1309: 83-94.

51. Tang G, Gudsnuk K, Kuo SH, Cotrina ML, Rosoklija G, Sosunov A, et al. Loss of mTOR-dependent macroautophagy causes autistic-like synaptic pruning deficits. Neuron. 2014;83(5):1131-43.

52. Zoghbi HY, Bear MF. Synaptic dysfunction in neurodevelopmental disorders associated with autism and intellectual disabilities. Cold Spring Harb Perspect Biol. 2012;4(3). https://doi.org/10.1101/cshperspect.a009886.

53. Gupta S, Ellis SE, Ashar FN, Moes A, Bader JS, Zhan J, et al. Transcriptome analysis reveals dysregulation of innate immune response genes and neuronal activity-dependent genes in autism. Nat Commun. 2014:5:5748.

54. Voineagu I, Wang $X$, Johnston P, Lowe JK, Tian Y, Horvath S, et al. Transcriptomic analysis of autistic brain reveals convergent molecular pathology. Nature. 2011;474(7351):380-4.

55. Gandal MJ, Haney JR, Parikshak NN, Leppa V, Ramaswami G, Hartl C, et al. Shared molecular neuropathology across major psychiatric disorders parallels polygenic overlap. Science. 2018:359(6376):693-7.

56. Lin YC, Frei JA, Kilander MB, Shen W, Blatt GJ. A subset of autismassociated genes regulate the structural stability of neurons. Front Cell Neurosci. 2016;10:263.

57. Borovac J, Bosch M, Okamoto K. Regulation of actin dynamics during structural plasticity of dendritic spines: signaling messengers and actinbinding proteins. Mol Cell Neurosci. 2018;91:122-30.

58. Maes M, Anderson G, Betancort Medina SR, Seo M, Ojala JO. Integrating autism spectrum disorder pathophysiology: mitochondria, vitamin A, CD38, oxytocin, serotonin and melatonergic alterations in the placenta and gut. Curr Pharm Des. 2019;25(41):4405-20. https://doi.org/10.2174/ 1381612825666191102165459.

59. Zhou W, Li S. Decreased levels of serum retinoic acid in chinese children with autism spectrum disorder. Psychiatry Res. 2018;269:469-73.

60. Carter MD, Shah CR, Muller CL, Crawley JN, Carneiro AM, VeenstraVanderWeele J. Absence of preference for social novelty and increased grooming in integrin beta3 knockout mice: initial studies and future directions. Autism Res. 2011:4(1):57-67.

61. Padmakumar M, Van Raes E, Van Geet C, Freson K. Blood platelet research in autism spectrum disorders: in search of biomarkers. Res Pract Thromb Haemost. 2019;3(4):566-77.

62. Ritvo ER, Yuwiler A, Geller E, Ornitz EM, Saeger K, Plotkin S. Increased blood serotonin and platelets in early infantile autism. Arch Gen Psychiatry. 1970; 23(6):566-72.

63. Bagni C, Zukin RS. A synaptic perspective of fragile $X$ syndrome and autism spectrum disorders. Neuron. 2019;101(6):1070-88.

64. Danesi C, Keinanen K, Castren ML. Dysregulated Ca(2+)-permeable AMPA receptor signaling in neural progenitors modeling fragile $X$ syndrome. Front Synaptic Neurosci. 2019:11:2.

65. Herman AB, Vrakas CN, Ray M, Kelemen SE, Sweredoski MJ, Moradian A, et al. FXR1 is an IL-19-responsive RNA-binding protein that destabilizes proinflammatory transcripts in vascular smooth muscle cells. Cell Rep. 2018, 24(5):1176-89.

66. Martinez-Cerdeno V. Dendrite and spine modifications in autism and related neurodevelopmental disorders in patients and animal models. Dev Neurobiol. 2017;77(4):393-404. 
67. Telias M. Molecular mechanisms of synaptic dysregulation in fragile $X$ syndrome and autism spectrum disorders. Front Mol Neurosci. 2019;12:51.

68. Zhang Z, Marro SG, Zhang Y, Arendt KL, Patzke C, Zhou B, et al. The fragile $X$ mutation impairs homeostatic plasticity in human neurons by blocking synaptic retinoic acid signaling. Sci Transl Med. 2018;10(452). https://doi.org/ 10.1126/scitransImed.aar4338.

69. Zhao X, Wang Y, Meng C, Fang N. FMRP regulates endothelial cell proliferation and angiogenesis via the miR-181a-CaM-CaMKII pathway. Cell Biol Int. 2018;42(10):1432-44.

70. Dean B. Understanding the pathology of schizophrenia: recent advances from the study of the molecular architecture of postmortem CNS tissue. Postgrad Med J. 2002;78(917):142.

71. Kathryn MG, Anthony AG. The role of neurotransmitters in schizophrenia Schizophrenia and psychotic spectrum disorders. Oxford: Oxford University Press; 2016. https://dx.doi.org/10.1093/med/9780199378067.003.0010.

72. Brown AS, Borgmann-Winter K, Hahn CG, Role L, Talmage D, Gur R, et al. Increased stability of microtubules in cultured olfactory neuroepithelial cells from individuals with schizophrenia. Prog Neuro-Psychopharmacol Biol Psychiatry. 2014;48:252-8.

73. Datta SR, McQuillin A, Rizig M, Blaveri E, Thirumalai S, Kalsi G, et al. A threonine to isoleucine missense mutation in the pericentriolar material 1 gene is strongly associated with schizophrenia. Mol Psychiatry. 2010;15(6): 615-28.

74. Gurling HM, Critchley H, Datta SR, McQuillin A, Blaveri E, Thirumalai S, et al Genetic association and brain morphology studies and the chromosome 8 p22 pericentriolar material 1 (PCM1) gene in susceptibility to schizophrenia. Arch Gen Psychiatry. 2006;63(8):844-54.

75. Hamshere ML, Walters JT, Smith R, Richards AL, Green E, Grozeva D, et al. Genome-wide significant associations in schizophrenia to ITIH3/4, CACNA1C and SDCCAG8, and extensive replication of associations reported by the schizophrenia PGC. Mol Psychiatry. 2013;18(6):708-12.

76. Moehle MS, Webber PJ, Tse T, Sukar N, Standaert DG, DeSilva TM, et al. LRRK2 inhibition attenuates microglial inflammatory responses. J Neurosci. 2012;32(5):1602-11.

77. Prabakaran S, Swatton JE, Ryan MM, Huffaker SJ, Huang JT, Griffin JL, et al. Mitochondrial dysfunction in schizophrenia: evidence for compromised brain metabolism and oxidative stress. Mol Psychiatry. 2004;9(7):684-97, 43.

78. Shimizu S, Matsuzaki S, Hattori T, Kumamoto N, Miyoshi K, Katayama T, et al. DISC1-kendrin interaction is involved in centrosomal microtubule network formation. Biochem Biophys Res Commun. 2008;377(4):1051-6.

79. Tzeng NS, Hsu YH, Ho SY, Kuo YC, Lee HC, Yin YJ, et al. Is schizophrenia associated with an increased risk of chronic kidney disease? A nationwide matched-cohort study. BMJ Open. 2015;5(1):e006777.

80. Tzur Bitan D, Krieger I, Berkovitch A, Comaneshter D, Cohen A. Chronic kidney disease in adults with schizophrenia: a nationwide population-based study. Gen Hosp Psychiatry. 2019;58:1-6.

81. Cao H, Chen J, Meyer-Lindenberg A, Schwarz E. A polygenic score for schizophrenia predicts glycemic control. Transl Psychiatry. 2017;7(12):1295.

82. Pillinger T, Beck K, Gobjila C, Donocik JG, Jauhar S, Howes OD. Impaired glucose homeostasis in first-episode schizophrenia: a systematic review and meta-analysis. JAMA Psychiatry. 2017;74(3):261-9.

83. Greenhalgh AM, Gonzalez-Blanco L, Garcia-Rizo C, Fernandez-Egea E, Mille $\mathrm{B}$, Arroyo MB, et al. Meta-analysis of glucose tolerance, insulin, and insulin resistance in antipsychotic-naïve patients with nonaffective psychosis. Schizophr Res. 2017;179:57-63.

84. Anney R, Klei L, Pinto D, Almeida J, Bacchelli E, Baird G, et al. Individual common variants exert weak effects on the risk for autism spectrum disorders. Hum Mol Genet. 2012;21(21):4781-92.

85. de la Torre-Ubieta L, Won H, Stein JL, Geschwind DH. Advancing the understanding of autism disease mechanisms through genetics. Nat Med. 2016;22:345.

86. Krumm N, O'Roak BJ, Karakoc E, Mohajeri K, Nelson B, Vives L, et al. Transmission disequilibrium of small CNVs in simplex autism. Am J Hum Genet. 2013:93(4):595-606.

87. Steinberg S, de Jong S, Mattheisen M, Costas J, Demontis D, Jamain S, et al. Common variant at 16p11.2 conferring risk of psychosis. Mol Psychiatry. 2014;19(1):108-14

88. Chang H, Li L, Li M, Xiao X. Rare and common variants at 16p11.2 are associated with schizophrenia. Schizophr Res. 2017;184:105-8.

\section{Publisher's Note}

Springer Nature remains neutral with regard to jurisdictional claims in published maps and institutional affiliations.
Ready to submit your research? Choose BMC and benefit from:

- fast, convenient online submission

- thorough peer review by experienced researchers in your field

- rapid publication on acceptance

- support for research data, including large and complex data types

- gold Open Access which fosters wider collaboration and increased citations

- maximum visibility for your research: over $100 \mathrm{M}$ website views per year

At BMC, research is always in progress.

Learn more biomedcentral.com/submissions 